\title{
The pharmacoeconomics of routine postoperative troponin surveillance to prevent and treat myocardial infarction after non-cardiac surgery
}

\author{
A Torborg, ${ }^{1} \mathrm{MB}$ ChB, FCA (SA); L Ryan, ${ }^{1,2} \mathrm{MB}$ ChB, FCA (SA); G Kantor, ${ }^{3,4}$ MB ChB, FRCP (Canada); \\ B M Biccard, ${ }^{1}$ MB ChB, FCA (SA), FFARCSI, MMedSci, PhD \\ ${ }^{1}$ Perioperative Research Group, Department of Anaesthetics, Nelson R Mandela School of Medicine, College of Health Sciences, \\ University of KwaZulu-Natal and Inkosi Albert Luthuli Central Hospital, Durban, South Africa \\ ${ }^{2}$ Department of Anaesthetics, Grey's Hospital, Pietermaritzburg, KwaZulu-Natal, South Africa \\ ${ }^{3}$ Department of Anaesthesiology, Faculty of Health Sciences, University of Cape Town, South Africa \\ ${ }^{4}$ Department of Anesthesiology and Perioperative Medicine, Case Western Reserve University, Cleveland, Ohio, USA
}

Corresponding author: B M Biccard (biccardb@ukzn.ac.za)

\begin{abstract}
Background. A postoperative troponin leak that was previously considered clinically insignificant has been independently associated with 30 -day mortality in unselected surgical patients $\geq 45$ years of age following non-cardiac surgery.

Objectives. To determine whether routine troponin surveillance following non-cardiac surgery and initiation of aspirin and statin therapy in troponin-positive patients is cost-effective.

Methods. Pharmacoeconomic analysis to determine the cost-effectiveness of routine postoperative surveillance for patients aged $\geq 45$ years undergoing non-cardiac surgery. We compared the total expected cost of hospital care of patients who received routine troponin surveillance and subsequent introduction of statin and aspirin therapy for 30 days in troponin-positive patients with the cost of hospital care of patients who did not receive troponin surveillance. We estimated a $25 \%$ relative risk reduction following statin and aspirin therapy for postoperative vascular mortality and non-fatal myocardial infarction.

Results. Routine troponin surveillance with initiation of aspirin and statin therapy was cost-effective, with an incremental cost of -R16 724 per event avoided.

Conclusion. Routine postoperative troponin surveillance in non-cardiac surgical patients $\geq 45$ years of age requiring a postoperative night in hospital is potentially cost-effective.
\end{abstract}

S Afr Med J 2014;104(9):619-623. DOI:10.7196/SAMJ.7654

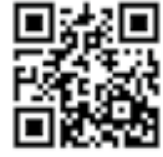

A postoperative troponin leak following noncardiac surgery is independently associated with 30-day mortality. ${ }^{[1]}$ Importantly, even what was previously considered an insignificant troponin leak (4th-generation troponin $\mathrm{T}>0.02 \mathrm{ng} / \mathrm{ml}$ ) has been independently associated with 30 -day mortality (odds ratio (OR) 2.41; 95\% confidence interval (CI) 1.33 - 3.77) in unselected patients aged $\geq 45$ years who underwent non-cardiac surgery. ${ }^{[1]}$ Over $40 \%$ of the population-attributable risk for 30-day mortality could be explained by the postoperative troponin leak. ${ }^{[1]}$

If generalisable, these data have wide-ranging public health implications. Firstly, of unselected patients aged $\geq 45$ years undergoing non-cardiac surgery, over $11 \%$ are expected to have a prognostically important postoperative troponin leak. ${ }^{[1]}$ Considering that over 200 million surgical procedures on adults are performed annually worldwide, ${ }^{[2]}$ and potentially half of these patients are $\geq 45$ years of age, it is expected that more than 5 million people will suffer a major perioperative cardiovascular event (i.e. cardiovascular mortality or myocardial infarction) within 30 days of surgery ${ }^{[1]}$ and over 10 million will have a postoperative troponin leak of prognostic importance. ${ }^{[1]}$

Should routine postoperative troponin surveillance be considered in this group of patients? Approximately a third of patients who have a postoperative troponin leak have a documented perioperative myocardial infarction. ${ }^{[3]}$ Targeting all patients with a troponin leak after non-cardiac surgery, since it indicates a myocardial injury, may be justified, as 30-day and intermediate-term mortality is significantly increased even in patients who do not fulfil study criteria for a perioperative myocardial infarction. ${ }^{[1,4,5]}$

Unfortunately there is no prospective randomised evidence of the utility of therapy for improving cardiovascular outcomes following a perioperative myocardial infarction. There are, however, good observational cohort data from a primary preventive trial of perioperative myocardial infarction showing that statins and aspirin given at the time of perioperative myocardial infarction were independently associated with decreased 30-day mortality, with adjusted ORs of 0.54 (95\% CI 0.29 - 0.99) and 0.26 (95\% CI 0.13 0.54), respectively. ${ }^{[6]}$ Whether statin and aspirin therapy may also decrease mortality in patients who sustain a postoperative troponin leak secondary to myocardial ischaemia (which is now known as myocardial injury after non-cardiac surgery (MINS)), without fulfilling the Universal Definition of Myocardial Infarction, ${ }^{[7,8]}$ is currently unknown.

Consideration of the economics of routine postoperative troponin surveillance is therefore warranted, as it is possible that the simple and inexpensive introduction of statin and aspirin therapy in patients who have a postoperative myocardial infarction may have a public health benefit, and should this benefit extend to MINS, it could be profound. 
The aim of this study was to determine whether routine troponin screening is cost-effective if a $25 \%$ relative risk reduction (RRR) in postoperative myocardial infarction and vascular mortality is achieved.

\section{Methods}

This pharmacoeconomic analysis determined the cost-effectiveness of routine postoperative surveillance for patients aged $\geq 45$ years undergoing non-cardiac surgery. ${ }^{[1]}$ It compared the total expected cost of hospital care of patients receiving routine troponin surveillance and subsequent introduction of statin and aspirin therapy for 30 days in troponin-positive patients with the cost of hospital care in patients who have not received troponin surveillance. We expected at least a $25 \%$ RRR in major cardiovascular complications (postoperative myocardial infarction and vascular death) at 30 days postoperatively should patients receive statin and aspirin therapy following a troponin leak. Based on the PeriOperative ISchemic Evaluation (POISE), trial we believed this to be a conservative estimate. ${ }^{[6]}$ Using either the upper CIs (smallest treatment effect) or the point estimates (expected treatment effect) of the cardiovascular protection associated with statin and aspirin in patients who had an infarction, one would expect an RRR for 30-day mortality of 0.53 ( 0.99 for statins $\times 0.54$ for aspirin) and 0.14 ( 0.54 for statins $\times 0.26$ for aspirin), respectively. ${ }^{[6]}$

From the Vascular events In noncardiac Surgery patIents cOhort evaluatioN (VISION) Study data, ${ }^{[1]}$ we determined the incidence of troponin-positive patients postoperatively (11.6\%) and the expected mortality (1.9\%). The expected incidence of cardiovascular complications associated with a troponin leak is shown in Fig. 1. In the VISION study, $45 \%$ of the deaths reported at 30 days were secondary to vascular causes. Vascular deaths were defined as deaths following a myocardial infarction, cardiac arrest, stroke, cardiac revascularisation procedure, pulmonary embolus or haemorrhage, or due to an unknown cause. ${ }^{[1]}$ We estimated that $37.3 \%$ of patients who have a postoperative troponin leak ${ }^{[6]}$ would have a clinical diagnosis of non-fatal myocardial infarction (3.96\% of the VISION cohort). Furthermore, we expected $73.4 \%$ of these complications to occur during the same admission for non-cardiac surgery, and the remaining $26.6 \%$ to occur after discharge but earlier than 30 days. ${ }^{[1]}$

Following initiation of statin and aspirin therapy in troponinpositive patients, we estimated a $25 \%$ RRR for vascular mortality and non-fatal myocardial infarction. We did not consider any efficacy associated with statin and aspirin therapy in patients who sustained MINS and did not fulfil the criteria for a perioperative myocardial infarction, as currently there are no data on the efficacy of these therapies in MINS.

All costs used were averages based on private healthcare costs in South Africa (SA). The costs of troponin surveillance were based on costs from two private laboratories. We considered the cost of troponin surveillance for the first 3 postoperative days, i.e. postoperative days 1, 2 and 3, and the cost of 30 days of low-dose aspirin (80 - $100 \mathrm{mg}$ daily) and statin (generic atorvastatin $40 \mathrm{mg}$ daily) therapy in patients who were troponin-positive. Average drug costs for aspirin and atorvastatin were obtained from Discovery Health. We used a drug dispensing fee of R25.

The costs of all adverse events used in this analysis were based on average Discovery Health costs for the period January 2011 - June 2011 for hospital admissions classified using Discovery's Diagnosis Related Groups (DRG) system (Table 1). We used the following costs: (i) the average hospital cost of all fatal myocardial infarctions was used for the cost of a fatal myocardial infarction occurring after hospital discharge; and (ii) the average hospital cost of a non-fatal myocardial infarction (without complications) was used for the attributable cost of a non-fatal myocardial infarction occurring after hospital discharge. The cost of in-hospital myocardial infarctions was calculated as $155 \%$ of the DRG average cost of a non-cardiac surgery hospital admission, based on the work of Dimick et al., ${ }^{[9]}$ which showed that in-hospital cardiovascular complications following noncardiac surgery increased adjusted expenditure by $155 \%$ compared with a non-cardiac surgical admission with minor complications. We only adjusted for the cost of the in-hospital complication, and not for duration of stay, as cardiovascular complications associated with surgery have not been associated with an increase in the duration of hospital stay. ${ }^{[10]}$

Screening for troponins will also result in increased subsequent cardiovascular risk stratification of patients. We therefore included the cost of three in-hospital screening electrocardiograms (ECGs) in order to diagnose a perioperative myocardial infarction, and four specialist cardiologist consultations (two inpatient and two outpatient visits), a full lipid profile, and measurement of glycated haemoglobin (HbAlc) for the expected proportion of diabetics in the troponin-positive cohort (based on the VISION data) ${ }^{[1]}$ for further risk stratification.

We constructed a monetary balance sheet in SA rands (ZAR) for the outcomes for a troponin surveillance group and a no-troponin surveillance group. All costs were calculated as the proportional cost per patient. For example, if $11.6 \%$ of patients were troponinpositive, the cost of treating troponin-positive patients with statins and aspirin would be $0.116 \times$ the cost of drug therapy. Similarly, the drug dispensing fee of R25 is therefore $0.116 \times$ R25 as a proportional cost per individual.

We also considered the cost-effectiveness of postoperative troponin surveillance. A common definition of cost-effectiveness is whether the average per capita contribution to the national gross domestic product (GDP) over a year is greater than the cost of a particular secondary preventive intervention. ${ }^{[11]}$ The average

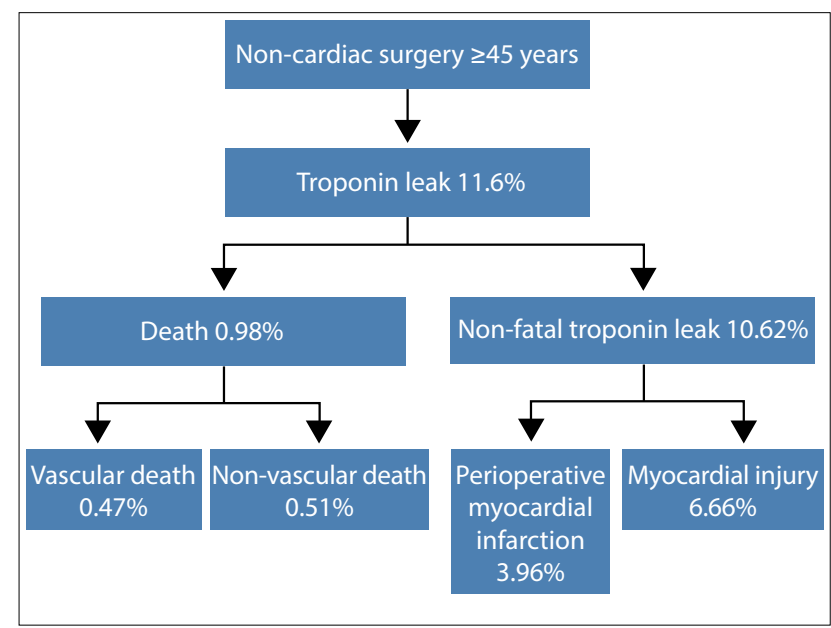

Fig. 1. Expected cardiovascular complications following a troponin leak in patients $\geq 45$ years of age undergoing non-cardiac surgery.

Table 1. Discovery Health's Diagnosis Related Groups (DRGs) used in the study

DRG 53310: acute myocardial infarction without complications and comorbidities

DRG 53320: acute myocardial infarction with complications and comorbidities

DRG 53330: acute myocardial infarction with major complications and comorbidities 
per capita contribution to the GDP in SA is approximately R49 134. ${ }^{[12]}$ By definition, therefore, a cost-effective intervention would cost less than R49 134 per event avoided.

\section{Results}

The average costs for troponin surveillance and the treatment of cardiovascular complications are shown in Table 2. The pharmacoeconomic analysis is shown in Table 3.

\section{Discussion}

This pharmacoeconomic analysis suggests that routine troponin surveillance in patients $\geq 45$ years of age undergoing non-cardiac surgery that requires a postoperative night in hospital is potentially cost-effective should statin and aspirin therapy decrease myocardial infarction and vascular mortality by $25 \%$. Routine troponin surveillance was associated with a R32 410 increase in cost per event avoided, which remains cost-effective as it is less than the annual per capita contribution to the GDP.

The fact that routine troponin surveillance is potentially cost-effective is important, as over $65 \%$ of patients who have a postoperative myocardial infarction are asymptomatic, ${ }^{[3,4]}$ and without troponin surveillance these cases will therefore be missed.

Although we have not considered any risk reduction associated with aspirin or statin therapy in patients with MINS in this pharmacoeconomic analysis, we would recommend that initiating these therapies after a diagnosis of MINS be considered in order to prevent a progression to myocardial infarction or vascular death. It is possible that these interventions may decrease subsequent cardiovascular morbidity in MINS patients who do not progress to myocardial infarction.

As a troponin leak is the largest contributor to 30-day postoperative mortality with a population-attributable risk of over $40 \%$, when considered with preoperative and surgical risk factors ${ }^{[1]}$ routine postoperative ECG surveillance would not be an acceptable substitute for troponin surveillance.

In conclusion, in the majority of cases the only way to identify patients who have had a myocardial infarction after non-cardiac surgery is to conduct routine postoperative troponin surveillance, and this is a potentially cost-effective intervention.

\section{Statin and aspirin therapy}

We did consider that introducing statin and aspirin therapy could increase drug-associated morbidity. The incidences of adverse events associated with postoperative statin and aspirin therapy are based on two individual data meta-analyses of aspirin and statin therapy. ${ }^{[13,14]}$ Statin therapy was not associated

Table 2. Average costs used in the pharmacoeconomic analysis

\begin{tabular}{ll}
\hline & Cost (ZAR) \\
\hline Troponin surveillance, drug costs and cardiovascular risk stratification & \\
Troponin surveillance (3 days) & 732.00 \\
Aspirin for 30 days & 18.26 \\
Atorvastatin for 30 days & 95.60 \\
ECG (three in hospital) & 237.90 \\
Full lipid profile & 250.50 \\
HbAlc & 131.75 \\
Cardiology consult & 379.95 \\
Cardiovascular complications & \\
Non-fatal myocardial infarction & 27684.26 \\
Fatal myocardial infarction & 59145.94 \\
Additional cost of an in-hospital cardiovascular complication & 70363.80 \\
ZAR = South African rands; ECG = electrocardiogram; HbAlc = glycated haemoglobin. &
\end{tabular}

Table 3. The cost per patient (in ZAR) of troponin* or no troponin surveillance in SA non-cardiac surgical patients $\geq 45$ years of age

\begin{tabular}{|c|c|c|}
\hline & $\begin{array}{l}\text { Troponin } \\
\text { surveillance }\end{array}$ & $\begin{array}{l}\text { No troponin } \\
\text { surveillance }\end{array}$ \\
\hline \multicolumn{3}{|c|}{ Direct and indirect costs of troponin surveillance and therapy (A) } \\
\hline Cost of troponin surveillance for 3 days & 732 & 0 \\
\hline Cost of statin and aspirin therapy for 30 days & 13.21 & 0 \\
\hline Drug dispensing fee & 2.9 & 0 \\
\hline \multicolumn{3}{|l|}{ Costs of perioperative cardiovascular complications (B) } \\
\hline Cost of fatal myocardial infarction & 581.15 & 660.32 \\
\hline $\begin{array}{l}\text { Cost of non-fatal myocardial infarction without } \\
\text { complications }\end{array}$ & 1988.76 & 2336.84 \\
\hline $\begin{array}{l}\text { Total cost per patient } \\
(C=A+B)\end{array}$ & $3318.02(\mathrm{C} 1)$ & $2997.16(\mathrm{C} 2)$ \\
\hline $\begin{array}{l}\text { Total incremental cost for surveillance and therapy } \\
(\mathrm{D}=\mathrm{C} 1-\mathrm{C} 2)\end{array}$ & 320.86 & \\
\hline Absolute risk reduction & 0.99 & \\
\hline NNT to prevent 1 event & 101 & \\
\hline $\begin{array}{l}\text { Total incremental cost } \\
(\mathrm{E}=\mathrm{D} \times \mathrm{NNT})\end{array}$ & 32409.80 & \\
\hline $\begin{array}{l}\text { Cost-effectiveness in SA patients } \\
(\mathrm{F}=\mathrm{E}-\mathrm{R} 49134)^{[11]}\end{array}$ & -16724.20 & \\
\hline
\end{tabular}

with significantly increased rhabdomyolysis in an individual patient data analysis of over 90000 patients from randomised controlled trials, ${ }^{[13]}$ and no adverse effects are therefore expected for statin therapy.

Aspirin has been shown to increase major gastrointestinal bleeding $(0.03 \%$ per year of therapy). ${ }^{[14]}$ Theoretically this could lead to a $0.0025 \%(0.03 \% / 12$ months) increase in gastrointestinal bleeding within 30 days of surgery, or a number-needed-to-harm of 40 000. A surgical meta-analysis suggests that the risk of postoperative bleeding is increased by a factor of 1.5 (median, interquartile range 1.0 - 2.5); however, only after transurethral prostatectomy (TURP) were there any deaths possibly attributable to bleeding. ${ }^{[15]}$ The issue of increased bleeding risk following TURP has been challenged by subsequent authors, and a randomised controlled trial evaluating the effect of lowdose acetylsalicylic acid on bleeding after TURP ${ }^{[16]}$ showed that while postoperative blood loss was increased in patients receiving 
aspirin therapy, there was no significant difference in operative blood loss, median time to catheter removal, length of hospital stay or the incidence of readmission to hospital due to secondary haemorrhage. As a result, the meta-analysis by Burger et al. ${ }^{[15]}$ makes no recommendations regarding perioperative aspirin use, and suggests that a controlled trial of surgical patients is needed.

The recently published PeriOperative Ischemic Evaluation-2 (POISE-2) trial ${ }^{[17]}$ did, however, show that aspirin given during surgery increased perioperative bleeding, and this risk remained until the 8th postoperative day. What is currently unclear is whether the initiation of aspirin therapy following a troponin leak in the postoperative period carries a similar bleeding risk to that in the POISE-2 trial. We would encourage vigilance once aspirin is initiated in the postoperative period, until such time as there are data to inform this issue.

We would still recommend initiation of aspirin therapy in patients who are troponin-positive postoperatively, as in secondary prevention trials aspirin decreases serious vascular events (numberneeded-to-treat (NNT) 67) and coronary events (NNT 100), ${ }^{[14]}$ and this is consistent with the observational data from the POISE trial in surgical patients. ${ }^{[3]}$ Troponin screening in an at-risk population and the initiation of aspirin therapy in those with increased troponin levels is therefore likely to be a cost-effective intervention.

There are a number of compelling reasons to believe that this pharmacoeconomic analysis may be conservative, and the benefits associated with routine troponin surveillance and statin and aspirin therapy may be larger than what is presented here.

Firstly, it is possible that routine troponin surveillance may also decrease subsequent morbidity and mortality associated with MINS through institution of appropriate treatment of underlying coronary artery disease, especially as MINS is associated with increased longterm cardiovascular morbidity. ${ }^{[5]}$ We would expect further benefit in patients with MINS, as these patients have been shown to have an increased risk of non-fatal cardiac arrest and subsequent coronary revascularisation. ${ }^{[6]}$ It has been shown in a meta-analysis of over 170000 patients that the addition of statin therapy significantly decreases subsequent coronary revascularisation, ${ }^{[18]}$ so there may be additional cost-saving benefits associated with treating postoperative myocardial injury that have not been calculated here.

Secondly, it is likely that statin and aspirin therapy may also decrease non-vascular mortality in addition to vascular mortality. A postoperative troponin leak is independently predictive of all-cause mortality, and this may be because an early postoperative myocardial injury often precedes further non-cardiac complications, aggravating their severity, which results in death. ${ }^{[1]}$ A postoperative troponin leak may therefore also be an initiating event in a non-vascular postoperative death. Importantly, and consistent with this conclusion, is that statins and aspirin were associated with a reduction in allcause mortality in the POISE study. ${ }^{[3]}$ It is therefore possible that if statin and aspirin administration decreased all-cause mortality and all myocardial injury after non-cardiac surgery by $25 \%$, there would be an overall cost saving with aspirin and statin therapy following routine troponin surveillance for non-cardiac surgery.

Thirdly, both statin and aspirin in secondary prevention trials have also decreased subsequent fatal and non-fatal stroke. ${ }^{[14,18]}$ This pharmacoeconomic analysis only considered fatal stroke, which is part of the definition of vascular mortality. ${ }^{[1]}$

It is also possible that the costs we have used in this pharmacoeconomic analysis are conservative. Davenport et al. ${ }^{[10]}$ have suggested that cardiovascular complications associated with surgery increase costs by $459 \%$ as opposed to the $155 \%$ used in this analysis, which would result in a cost saving associated with routine troponin surveillance of R16 700 per event avoided in our analysis.
Furthermore, the predicted costs of treating myocardial infarctions in 2011 across all medical aid providers was R78 $869,{ }^{[19]}$ which is higher than the Discovery Health costs for the same period used in this analysis. If the average cost of all medical aid providers was used in our model, there would have been an absolute cost saving per event avoided. Both these possible scenarios change this intervention from a cost-effective intervention to a cost-saving intervention.

An outcome that may change the cost-effectiveness of this pharmacoeconomic analysis is out-of-hospital sudden death, which is associated with no additional cost. When we considered this scenario for all out-of-hospital deaths in the analysis, routine troponin screening remained cost-effective.

Finally, it is important that the finding of a postoperative troponin leak does not lead to an unnecessary increase in expenditure associated with subsequent inappropriate coronary angiography investigation. We would advise against routine coronary angiography if the patient does not fulfil current accepted indications for this procedure. This is because significant differences exist between spontaneous (medical) myocardial infarctions and myocardial infarction following surgery. ${ }^{[20]}$ The postoperative patient is exposed to an environment associated with haemodynamic instability, procoagulation, sympathetic stress and potential bleeding and hypoxia, ${ }^{[21]}$ and as such the pathophysiology of the perioperative myocardial infarction is likely to be different to the myocardial infarction characteristic of medical patients. ${ }^{[22]}$ These factors may partly explain the predominance of ST-segment depression with surgical myocardial infarctions. ${ }^{[3,23]} \mathrm{We}$ would recommend a conservative approach to coronary angiography in patients with a postoperative troponin leak, until such time as the coronary computed tomography angiography data from the VISION study are published. ${ }^{[24]}$ Furthermore, as there are no prospective studies on managing perioperative myocardial infarctions and MINS, the current recommendations for coronary angiography in these patients include very specific scenarios that probably occur in a very small group of patients (in the region of $2-3 \%$ of patients who have a documented myocardial infarction). ${ }^{[25,26]}$

Finally, although our pharmacoeconomic analysis was conducted for troponin surveillance of all non-cardiac surgical patients $\geq 45$ years of age requiring an overnight hospital stay, the recently published MINS study showed that low-risk surgery was independently protective for the development of MINS (hazard ratio 0.72; 95\% CI 0.55 - 0.99) ${ }^{[7]} \mathrm{We}$ would therefore not recommend routine troponin surveillance for lowrisk surgical procedures as defined in the VISION study.

\section{Study limitations}

There are limitations that may affect the accuracy of this pharmacoeconomic analysis. The analysis was conducted without consideration of potential postoperative troponin surveillance that is already conducted following some high-risk surgical procedures. This would not change the outcome of this analysis, as we therefore overestimated the cost of this suggested change to routine troponin surveillance. However, the importance of our analysis is that it suggests that extending the scope of troponin surveillance is potentially cost-effective, provided effective therapy is immediately provided when a troponin leak is detected.

Furthermore, there are no data indicating that interventions for MINS decrease subsequent major adverse cardiac events in low-risk patients. We therefore cannot include this analysis in the pharmacoeconomic analysis, although it is an area that may be associated with a large public health benefit, should efficacy be shown in these patients. This is an area of perioperative medicine that requires urgent investigation. Based on the increased mortality and cardiovascular morbidity, ${ }^{[5,7]}$ we would recommend considering these patients for subsequent secondary prevention for coronary artery disease. 


\section{Conclusion}

This pharmacoeconomic analysis suggests that routine postoperative troponin surveillance is a potentially cost-effective intervention in all patients $\geq 45$ years of age undergoing inpatient non-cardiac surgery.

\section{References}

1. Devereaux PJ, Chan MT, Alonso-Coello P, et al. Association between postoperative troponin levels and 30day mortality among patients undergoing noncardiac surgery JAMA 2012:307(21):2295-304. [http://dx do org/10.1001/jama.2012.5502

2. Weiser TG, Regenbogen SE, Thompson KD, et al. An estimation of the global volume of surgery: $\mathrm{A}$ modelling strategy based on available data. Lancet 2008;372(9633):139-144. [http://dx.doi.org/10.1016/S01406736(08)60878-8]

3. Devereaux PJ, Xavier D, Pogue J, et al. Characteristics and short-term prognosis of perioperative myocardial infarction in patients undergoing noncardiac surgery: A cohort study. Ann Intern Med 2011;154(8):523-528. [http://dx.doi.org/10.1059/0003-4819-154-8-201104190-00003]

4. Van Waes JA, Nathoe HM, de Graaff JC, et al. Myocardial injury after noncardiac surgery and its association with short-term mortality Circulation 2013:127(23):2264-2271. [http://dxdoiorg/10.1161/ CIRCULATIONAHA.113.002128

5. Levy M, Heels-Ansdell D, Hiralal R, et al. Prognostic value of troponin and creatine kinase muscle and brain isoenzyme measurement after noncardiac surgery: A systematic review and meta-analysis. Anesthesiology 2011;114(4):796-806. [http://dx.doi.org/10.1097/ALN.0b013e31820ad503]

6. Devereaux PJ, Xavier D, Pogue I, et al. Characteristics and short-term prognosis of perioperative myocardial infarction in patients undergoing noncardiac surgery: A cohort study. Ann Intern Med 2011;154(8):523-528. [http://dx.doi.org/10.1059/0003-4819-154-8-201104190-00003]

7. Botto F, Alonso-Coello P, Chan MT, et al. Myocardial injury after noncardiac surgery: A large, international, prospective cohort study establishing diagnostic criteria, characteristics, predictors, and 30-day outcomes. Anesthesiology 2014;120(3):564-578. [http://dx.doi.org/10.1097/ALN.0000000000000113]

8. Thygesen K, Alpert IS, Jaffe AS, Simoons ML, Chaitman BR, White HD. Third Universal Definition of Myocardial Infarction. J Am Coll Cardiol 2012;60(16):1581-1598 [http://dx.doi.org/10.1016/j.jacc.2012 08.001] 9. Dimick JB, Chen SL, Taheri PA, Henderson WG, Khuri SF, Campbell DA Ir. Hospital costs associated with surgical complications: a report from the private-sector National Surgical Quality Improvement Program. Am Coll Surg 2004;199(4):531-537. [http:///dx.doi.org/10.1016/j.jamcollsurg.2004.05.276]

10. Davenport DL, Henderson WG, Khuri SF, Mentzer RM Jr. Preoperative risk factors and surgical complexity are more predictive of costs than postoperative complications: A case study using the National Surgical Quality Improvement Program (NSQIP) database. Ann Surg 2005;242(4):463-438; discussion 468-471. [http://dx.doi org/10.1097/01.sla.0000183348]

11. Ortegon M, Lim S, Chisholm D, Mendis S. Cost effectiveness of strategies to combat cardiovascular disease diabetes, and tobacco use in sub-Saharan Africa and South East Asia: Mathematical modelling study. BM] 2012;344:e607. [http://dx.doi.org/10.1136/bmj.e607]

12. Department of Health, South Africa. Millenium Development Goals. Goal 8: Develop a Global Partnership for Health. http://wwwwstatssa.gov.za/nss/Goal_Reports/GOAL\%208-DEVELOP\%20A\%20GLOBAL\%20 PARTNERSHIP\%20FOR\%20DEVELOPMENT1.pdf (accessed 16 April 2014).
13. Baigent C, Keech A, Kearney PM, et al. Efficacy and safety of cholesterol-lowering treatment: Prospective meta-analysis of data from 90,056 participants in 14 randomised trials of statins. Lancet 2005;366(9493):12671278. [http://dx.doi.org/10.1016/S0140-6736(05)67394-1]

14. Baigent C, Blackwell L, Collins R, et al. Aspirin in the primary and secondary prevention of vascular disease: Collaborative meta-analysis of individual participant data from randomised trials. Lancet 2009;373(9678):1849-1860. [http://dx.doi.org/10.1016/S0140-6736(09)60503-1]

15. Burger W, Chemnitius IM, Kneissl GD, Rucker G. Low-dose aspirin for secondary cardiovascular prevention - cardiovascular risks after its perioperative withdrawal versus bleeding risks with its continuation - review and meta-analysis. J Intern Med 2005;257(5):399-414. [http://dx.doi.org/10.1111/j.1365-2796.2005.01477.x]

16. Nielsen JD, Holm-Nielsen A, Jespersen J, Vinther CC, Settgast IW, Gram J. The effect of low-dose acetylsalicylic acid on bleeding after transurethral prostatectomy - a prospective, randomized, double-blind, placebocontrolled study. Scand J Urol Nephrol 2000;34(3):194-198.

17. Devereaux PJ, Mrkobrada M, Sessler DI, et al. Aspirin in patients undergoing noncardiac surgery. N Engl J Med 2014;370 (16):1494-503. [http://dx.doi.org/10.1056/NEJMoa1401105]

18. Baigent C, Blackwell L, Emberson I, et al. Efficacy and safety of more intensive lowering of LDL cholesterol: A meta-analysis of data from 170,000 participants in 26 randomised trials. Lancet 2010;376(9753):1670-1681. [http://dx.doi.org/10.1016/S0140-6736(10)61350-5]

19. Bergh M, Marais CA, Miller-Janson H, Salie F, Stander MP. Economic appraisal of dabigatran as first-line herapy for stroke prevention in atrial fibrillation. S Afr Med J 2013;103(4):241-245. [http://dx.doi.org/10.7196/ samj.6471

20. Ryan L, Rodseth RN, Biccard BM. The treatment of perioperative myocardial infarctions following noncardiac surgery. Southern African Journal of Anaesthesia and Analgesia 2012;18(2):86-93. [http://reference.sabinet co.za/webx/access/electronic_journals/medsajaa/medsajaa_v18_n2_a4.pdf

21. Devereaux PJ, Goldman L, Cook DJ, Gilbert K, Leslie K, Guyatt GH. Perioperative cardiac events in patients undergoing noncardiac surgery: A review of the magnitude of the problem, the pathophysiology of the events and methods to estimate and communicate risk. CMAJ 2005;173(6):627-634. [http://dxdoiorg/10.1503/ cmaj.050011

22. Biccard BM, Rodseth RN. The pathophysiology of perioperative myocardial infarction. Anaesthesia 2010;65(7):733-741. [http://dx.doi.org/10.1111/j.1365-2044.2010.06338.x.]

23. Landesberg G, Shatz V, Akopnik I, et al. Association of cardiac troponin, CK-MB, and postoperative myocardial ischemia with long-term survival after major vascular surgery. J Am Coll Cardiol 2003;42(9):15471554. [http://dx.doi.org/10.1016/j.jacc.2003.05.001] 24. Sheth T, Butler C, Chow B, et al. The coronary CT angiography vision protocol: A prospective observational
imaging cohort study in patients undergoing non-cardiac surgery. BMJ Open 2012;2(4):e001474. [http:// dx.doi.org/10.1136/bmjopen-2012-001474]

25. Biccard BM. Detection and management of perioperative myocardial ischemia. Curr Opin Anaesthesiol 2014;27(3):336-43. [http://dx.doi.org/10.1097/ACO.0000000000000071]

26. Biccard BM. Peri-operative myocardial infarction. Southern African Journal of Anaesthesia and Analgesia 2010;16(1):44-46.

Accepted 30 April 2014 